\title{
CHOOSING OF OPTIMAL REFERENCE SAMPLES FOR BOREAL LAKE CHLOROPHYLL A CONCENTRATION MODELING USING AERIAL HYPERSPECTRAL DATA
}

\author{
A.- L. Erkkilä̈, ${ }^{\mathrm{a},}$ I. Pölönen ${ }^{\mathrm{a}}$, A. Lindfors ${ }^{\mathrm{b}}$, E. Honkavaara ${ }^{\mathrm{c}}$, K. Nurminen ${ }^{\mathrm{c}}$, R. Näsi ${ }^{\mathrm{c}}$ \\ ${ }^{a}$ Faculty of Information Technology, University of Jyväskylä, PO Box 35, FI-40014 Jyväskylä, Finland, \\ (anna-leena.j.erkkila, ilkka.polonen)@jyu.fi \\ ${ }^{\mathrm{b}}$ Luode Consulting Oy, Sinimäentie 10 B, FI-02630, Finland \\ ${ }^{c}$ Finnish Geospatial Research Institute FGI, Geodeetinrinne 2, FI-02430 Masala, Finland
}

Commission III, WG III/4

KEY WORDS: Aerial remote sensing, lake water color, water quality monitoring, hyperspectral imaging, chlorophyll a

\begin{abstract}
:
Optical remote sensing has potential to overcome the limitations of point estimations of lake water quality by providing spatial and temporal information. In open ocean waters the optical properties are dominated by phytoplankton density, while the relationship between color and the constituents is more complicated in inland waters varying regionally and seasonally. Concerning the difficulties relating to comprehensive modeling of complex inland and coastal waters, the alternative approach is considered in this paper: the raw digital numbers (DN) recorded using aerial remote hyperspectral sensing are used without corrections and derived by means of regression modeling to predict Chlorophyll a (Chl-a) concentrations using in situ reference measurements. The target of this study is to estimate which number of local reference measurements is adequate for producing reliable statistical model to predict Chl-a concentration in complex lake water ecosystem. Based on the data collected from boreal lake Lohjanjärvi, the effect of standard deviation of Chl-a concentration of reference samples and their local clustering on predictability of model increases when number of reference samples or bands used as model variables decreases. However, the 2 or 3 band models are beneficial and more cost efficient when compared to 5 or 7 band models when the standard deviation of Chl-a concentration of reference samples is over certain level. The simple empirical approach combining remote sensing and traditional sampling may be feasible for regional and seasonal retrieval of Chl-a concentration distributions in complex ecosystems, where the comprehensive models are difficult or even impossible to derive.
\end{abstract}

\section{INTRODUCTION}

The simple single-variable algorithms consisting bands in the blue to green region are appropriate for Chlorophyl a (Chl-a) retrieval for vast areas of oceanic Case- 1 waters. These models fails in predicting the optically multicomponent Case- 2 water systems, such as coastal and inland waters, in which the Chl-a, colored dissolved organic matter (CDOM) and total suspended matter (TSM) may vary independently of each other. In addition, the relationship between color and the constituents of Case- 2 water may vary regionally and seasonally and there may be other affecting factors as reflectance from bottom in shallow water areas. The atmospheric correction procedures for inland water remote sensing are also challenging when compared to oceanic measurements. The climate changes may also change dynamic interactions between components and algorithms based on data of the past decades might turn out to be inaccurate in the near future. During last decades the investigation of optically complex Case-2 water bodies have received increasing attention. Besides the simple algorithms utilizing combinations of radiance or reflectance of few bands to estimate various lake water properties, the advanced neural network, semi-analytical and bio-optical modeling inversion methods have been developed to predict inherent optical properties (IOPs), (see, e.g. (IOCCG, 2006), (Palmer et al., 2015)).

Ocean color sensors carried by satellites have usually limited number of wavelength bands and therefore the methods that utilize

\footnotetext{
${ }^{*}$ Corresponding author
}

full hyperspectral data are mainly developed using airborne and hand-held sensors. Full measured reflectance spectrum is compared to the libraries of modeled spectra for simultaneous determining of Chl-a, CDOM and suspended matter concentrations e.g. in (Kutser et al., 2001). The algorithms for determination the inland optical components are usually validated only a limited range of lakes; global scale studies are needed for more robust and comprehensive models ((Bukata, 2013), (Palmer et al., 2015)). In this study, oppositely to attempt for comprehensive model, the goal is to study the applicability and reliability of use of raw digital numbers (DNs) recorded by means of aerial remote hyperspectral sensing to estimate Chl-a concentration in lake water. Representativity of limited number of local measurements for calibration of a simple statistical regression model, applied to whole area of a single lake, is evaluated.

\section{MATERIALS AND METHODS}

\subsection{Study area and measurements}

The remote sensing investigations of boreal lake Lohjanjärvi were executed with novel framing hyperspectral imager, which uses piezo-actuated Fabry-Perot interferometer (FPI) (Mäkynen et al., 2012). First prototypes of these imagers have been used in several different applications for environmental remote sensing (see, e.g, (Pölönen et al., 2012), (Honkavaara et al., 2013), (Näsi et al., 2015), (Honkavaara et al., 2016)) and to detect skin cancer (Pölönen, 2013). Newest version of spectral imagers using FPI are commercially available (http://www.rikola.fi). In this study, 


\begin{tabular}{|l|l|l|l|}
\hline Bands & RMSE $(\mu \mathrm{g} / \mathrm{l})$ & $\mathrm{r}-2$ & $\mathrm{r}$ \\
\hline 2 & 2.42 & 0.719 & 0.848 \\
\hline 3 & 2.17 & 0.773 & 0.879 \\
\hline 5 & 2.13 & 0.784 & 0.885 \\
\hline 7 & 2.11 & 0.788 & 0.888 \\
\hline 36 & 2.01 & 0.815 & 0.903 \\
\hline
\end{tabular}

Table 1. Statistics of the regression models: Root mean square error (RMSE), correlation coefficient $r$ and coefficient of determination $\mathrm{r}^{2}$.

the prototype $2012 \mathrm{~b}$ was used to capture VIS/NIR spectral range images comprised from 36 wavelength bands from $500 \mathrm{~nm}$ to $875 \mathrm{~nm}$. FPI images were captured from a manned single engine aircraft Cessna 172 Reims Rocket with an FPI camera using a flight height of about $2025 \mathrm{~m}$ above the mean sea level, providing a GSD of $2 \mathrm{~m}$. Block consisted of a total of 14 flight strips and 622 images. Forward overlaps were $75 \%$ and side overlaps were $53 \%$ in average. In situ reference variables of water quality were measured from a moving research vessel and seven water samples were taken for laboratory analysis for the calibration of the field data. The parameters measured were typical descriptors of eutrophication or otherwise important key elements of aquatic communities and health (Chl-a, BGA, NO23-N, Turbidity, TOC, NO23-N). Reference values of Chl-a consists of 650 measurements from different basins of Lohjanjärvi $\left(\mathrm{A}=89 \mathrm{~km}^{2}\right)$ with sampling distance varying for 5 to 15 meter depending on vessel speed. Detailed description of measuring procedure is presented in (Erkkilä et al., 2017).

\subsection{Data and algorithms}

The range, mean and standard deviation of 650 measured Chl-a reference values are $1.93-26.06 \mu \mathrm{g} / \mathrm{l}, 11.46 \mu \mathrm{g} / \mathrm{l}$ and $4.56 \mu \mathrm{g} / \mathrm{l}$, respectively. Multiple linear regression was used in deriving the model for predicting concentration of Chl-a by combination of DNs of different wavelengths measured by aerial hyperspectral imaging. The relation between measured Chl-a and regression estimated using all available 36 bands is presented in Figure 1. The combinations of 2, 3, 5 or 7 bands were selected using maximum correlation with Chl-a concentration as an criterion; peak wavelengths for the best 2 bands combination are 693 and 850 nm, for 3 bands: 567,682 and $850 \mathrm{~nm}$, for 5 bands : 540, 567, 636, 682 and $850 \mathrm{~nm}$ and for 7 bands: 540, 567, 636, 662, 682, 733 and $850 \mathrm{~nm}$. The basic statistics of the regression models applied to total 650 reference samples are presented in Table 1 . Different numbers $(8,10,12,15,20$ and 30) of randomly selected Chl-a concentration samples were used as calibration subset in deriving the regression models and then models are applied to the rest of measurements used as validation subset. 50000 randomly selected combinations without duplicates were taken for each number of reference Chl-a samples. The standard deviation of Chl-a concentrations and average minimum distance of location of each selected combinations were calculated. The average minimum distance is derived by determining minimum distance of each member of one selection from other members belonging to the same selection and then taking an average of these minimum distances of members. Two dimensional probability and cumulative probability histograms were determined for presenting and comparison of results.

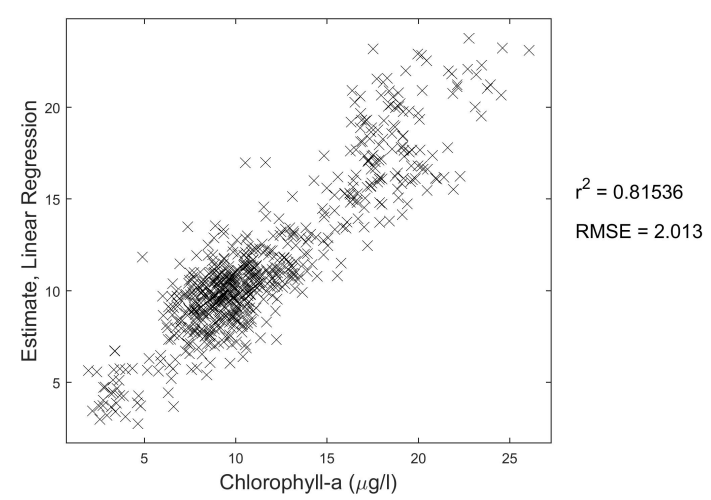

Figure 1. Linear regression estimate of Chlorophyll a concentration when DNs of all bands are used as variables.

\section{RESULTS}

50000 numbers of 12 randomly selected reference Chl-a observations have been used for deriving the regression model using the DN of 5 bands as an variables. The measured Chl-a concentration at all other of 650 reference location were considered as validation subset. The correlation coefficient $r$ (Figures 2 and 4) is determined between the validation subset and Chl-a concentration predicted by derived model at corresponding locations. Four examples out of 50000 tests are illustrated in Figure 3. The $r$ of all 50000 tests are presented as a function of standard deviation of Chl-a concentration of each 12 random samples by a scatter plot and two dimensional histogram in Figure 2. Probability histogram and cumulative probability histogram with respect to $r$ are presented in Figure 4. Only standard deviation bins, in which the sum of counts is over 30 , are plotted. The cumulative histogram is presented for RMSE and correlation coefficient and RMSE are also presented as a function of average minimum distance parameter describing the clustering of locations of 12 reference samples. Correlation coefficients and errors at different cumulative probability levels, $0.01,0.05,0.1,0.2$ and 0.5 , are presented in Figure 6 . The higher standard deviation improves the correlation coefficient but does not ensure low RMSE; see an example presented in Figure 3 (bottom left), where the correlation is fairly high $(r=0.77)$, but there is bias in the slope of predicted values, which explains the high error. Neither the high standard deviation nor large average minimum distance guarantee good predictability of the model with $99 \%$ reliability level (probability $=0.01$ ), when 12 reference samples are used in deriving the model with $\mathrm{DN}$ of 5 bands. However the situation improves and is stabilized significantly with reliability level $95 \%$ if the standard deviation of reference values is adequately high.

The effect of number of reference values are presented by cumulative probability histograms in Figure 7 and by correlation coefficient and RMSE at reliability level $95 \%$ as a function of standard deviation and average minimum distance in Figure 8. The $r$ and RMSE approach toward extreme values presented in Table 1 when number of reference samples increases. The 30 reference samples provide good predictability of model even independently of the standard deviation and average minimum distance levels studied. Decrease in range of standard deviation levels caused by the higher number of reference samples may also have an effect on the observed independence. 
The effect of number of independent variables on the $r$ and RMSE is presented in Figures 9 and 10. With 30 reference locations, 2 and 3 band models approach their extreme values for $r$ and RMSE presented in Table 1. They also require lower amount of reference measurements to produce reliable model, but the result is more sensitive to standard deviation and clustering of reference samples than when DN of 5 or 7 bands are used. Probability to reach at least $r=0.7$ is presented in Figure 11. For 5 bands model the $r$ at least 0.7 is almost certain when 30 reference measures are used. If 12 reference samples are used, the probability is significantly dependent on standard deviation of reference values. With 2 and 3 band models the $r$ at least 0.7 and RMSE lower than $4 \mu \mathrm{g} / \mathrm{l}$ are achieved almost certainly if standard deviation of 12 reference measurements is roughly over $4.5 \mu \mathrm{g} / 1$ (Figures 11 and 12).
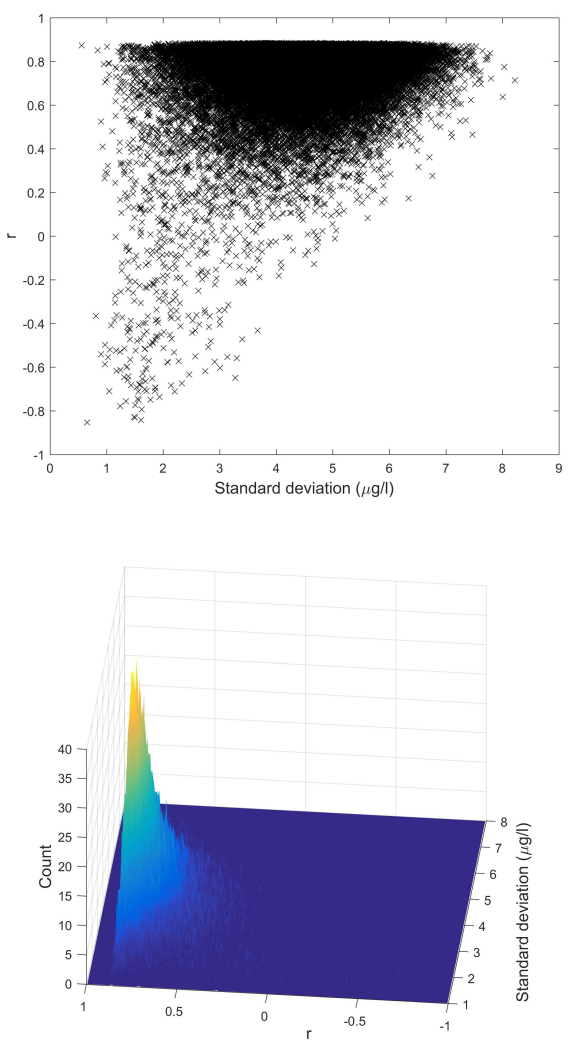

Figure 2. Correlation coefficient between Chl-a estimates of linear regression model and in-situ Chl-a measurements. Model is derived using 12 randomly selected reference $\mathrm{Chl}-\mathrm{a}$ measurements (50000 random selections) and DN of five bands as variables. Result is presented as a function of standard deviation of Chl-a concentration of 12 reference samples. Top: scatter plot. Bottom: 2D histogram.

\section{DISCUSSION AND CONCLUSION}

In most studied cases the variation of correlations and errors depends only slightly on the clustering or variability of the reference measurements. The lower number of reference measurements or bands used in model increases this dependency. The effect is highest when correlation coefficient is studied as a function of standard deviation of reference measurements, while RMSE does not express equally notable trend. As can be expected, the higher number of observations (reference samples) improve the
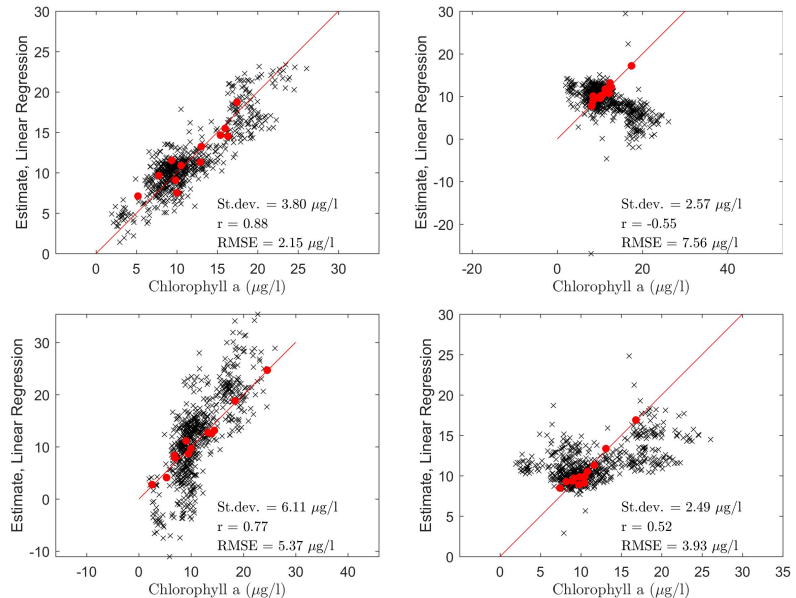

Figure 3. Four examples of comparison of reference Chl-a concentrations and estimates derived by regression model based on Chl-a measurements from 12 random locations (red circles and St.dev. values) are presented. The model is applied to the rest 638 Chl-a samples (black crosses, $r$ and RMSE values).
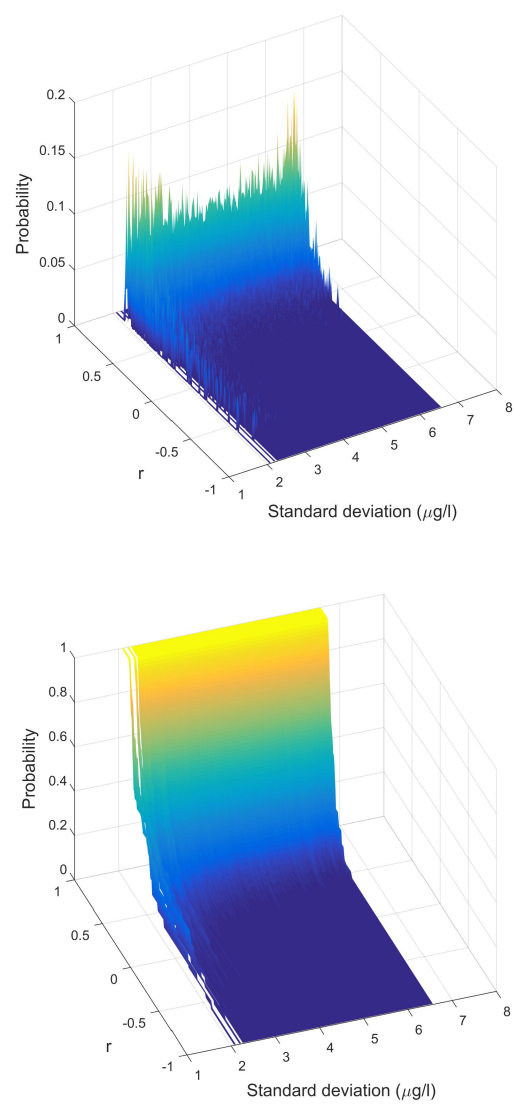

Figure 4. Probability histogram (top) and cumulative probability histogram (bottom) derived from data presented in Figure 1. The standard deviation bins for which the sum of counts is below 30 are rejected from probability histograms.

model predictability. The more prominent observation was, that the higher number of input variables (wavelength bands) in model would require significantly more reference measurements to approach the same predictability as model with lower number of input variables. Roughly, the 7 bands model requires 30 refer- 

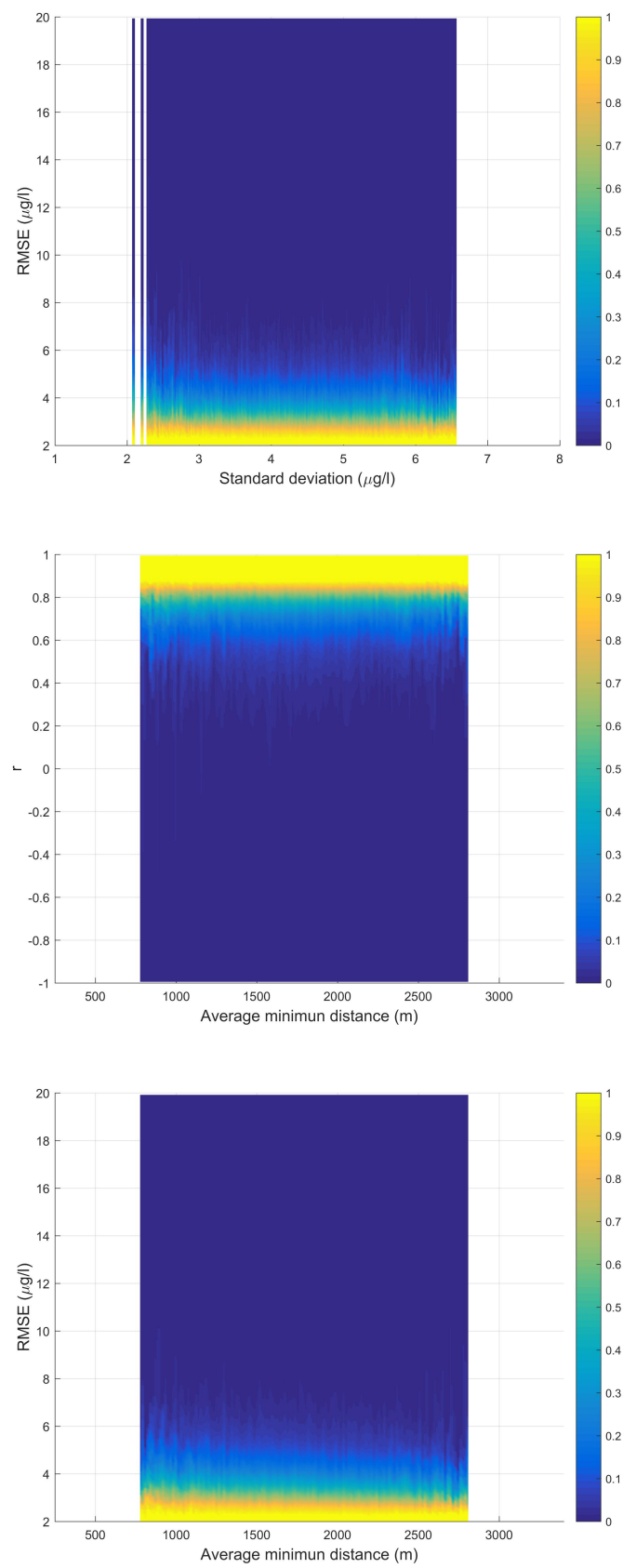

Figure 5. Cumulative probability histograms derived using 5 bands and 12 randomly selected reference samples. The standard deviation bins (top) or average minimum distance bins (middle and bottom) for which the sum of counts is below 30 are rejected from probability histograms.

ence samples to achieve same correlation coefficient with same confidence level as 2 or 3 band model reach with 12 reference measurements if their standard deviation of Chl-a concentrations is at least $4.5 \mu \mathrm{g} / \mathrm{l}$. The observations based on the empirical data of this study implies that better cost efficiency can be attained with 2 or 3 bands usage instead of 5 or 7 bands, when the amount of reference samples are wished to minimized. The more comprehensive multi-criteria optimization could be used to estimate a more accurate cost minimum. It would also be interesting to test the same approach with other lake data (different lake or same lake at different seasons) to see if the trends and optima would be the same; other optically active water quality parameters can be studied as well. The simple empirical approach combining remote sensing and traditional sampling methods may prove to be feasible when the goal is accurate long term monitoring of complex ecosystems.

\section{ACKNOWLEDGEMENT}

Study was partly funded by University of Jyvskyl and Tekes, the Finnish Funding Agency for Innovation (grants: 2208/31/2013 and $1711 / 31 / 2016$ )

\section{REFERENCES}

Bukata, R. P., 2013. Retrospection and introspection on remote sensing of inland water quality:like déjà vu all over again. Journal of Great Lakes Research 39, pp. 2-5.

Erkkilä, A.-L., Lindfors, A., Pölönen, I., Honkavaara, E., Nurminen, K., Näsi, R. and Ojanen, H., 2017. Water quality estimation in boreal lake by novel framing hyperspectral imaging. Submitted to Remote sensing of environment.

Honkavaara, E., Eskelinen, M. A., Pölönen, I., Saari, H., Ojanen, H., Mannila, R., Holmlund, C., Hakala, T., Litkey, P., Rosnell, T. et al., 2016. Remote sensing of 3-d geometry and surface moisture of a peat production area using hyperspectral frame cameras in visible to short-wave infrared spectral ranges onboard a small unmanned airborne vehicle (uav).

Honkavaara, E., Saari, H., Kaivosoja, J., Pölönen, I., Hakala, T., Litkey, P., Mäkynen, J. and Pesonen, L., 2013. Processing and assessment of spectrometric, stereoscopic imagery collected using a lightweight uav spectral camera for precision agriculture. Remote Sensing 5(10), pp. 5006-5039.

IOCCG, 2006. Remote Sensing of Inherent Optical Properties: Fundamentals, Tests of Algorithms, and Applications. Reports of the International Ocean Colour Coordinating Group, Vol. No. 5, IOCCG, Dartmouth, Canada.

Kutser, T., Herlevi, A., Kallio, K. and Arst, H., 2001. A hyperspectral model for interpretation of passive optical remote sensing data from turbid lakes. Science of the Total Environment 268(1), pp. 47-58.

Mäkynen, J., Saari, H., Holmlund, C., Mannila, R. and Antila, T., 2012. Multi-and hyperspectral uav imaging system for forest and agriculture applications. In: SPIE Defense, Security, and Sensing, International Society for Optics and Photonics, pp. 837409837409.

Näsi, R., Honkavaara, E., Lyytikäinen-Saarenmaa, P., Blomqvist, M., Litkey, P., Hakala, T., Viljanen, N., Kantola, T., Tanhuanpää, T. and Holopainen, M., 2015. Using uav-based photogrammetry and hyperspectral imaging for mapping bark beetle damage at tree-level. Remote Sensing 7(11), pp. 15467-15493.

Palmer, S. C., Kutser, T. and Hunter, P. D., 2015. Remote sensing of inland waters: Challenges, progress and future directions. Remote Sensing of Environment 157, pp. 1 - 8.

Pölönen, I., 2013. Discovering knowledge in various applications with a novel hyperspectral imager. Jyväskylä studies in computing; 1456-5390; 184.

Pölönen, I., Salo, H., Saari, H., Kaivosoja, J., Pesonen, L. and Honkavaara, E., 2012. Biomass estimator for nir image with a few additional spectral band images taken from light uas. In: SPIE Defense, Security, and Sensing, International Society for Optics and Photonics, pp. 836905-836905. 

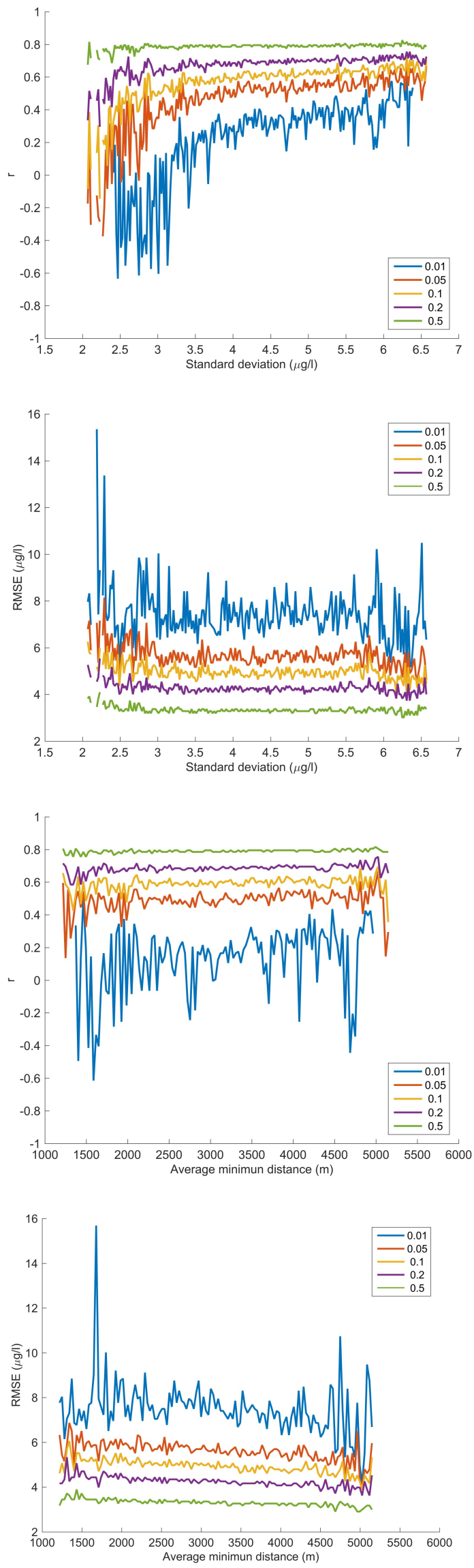

Figure 6. Correlation coefficient $r$ (top and second from bottom) and root mean square error (RMSE) (second from top and bottom) for different probability levels. All figues: 12 randomly selected reference samples, 50000 random selection and 5 bands.
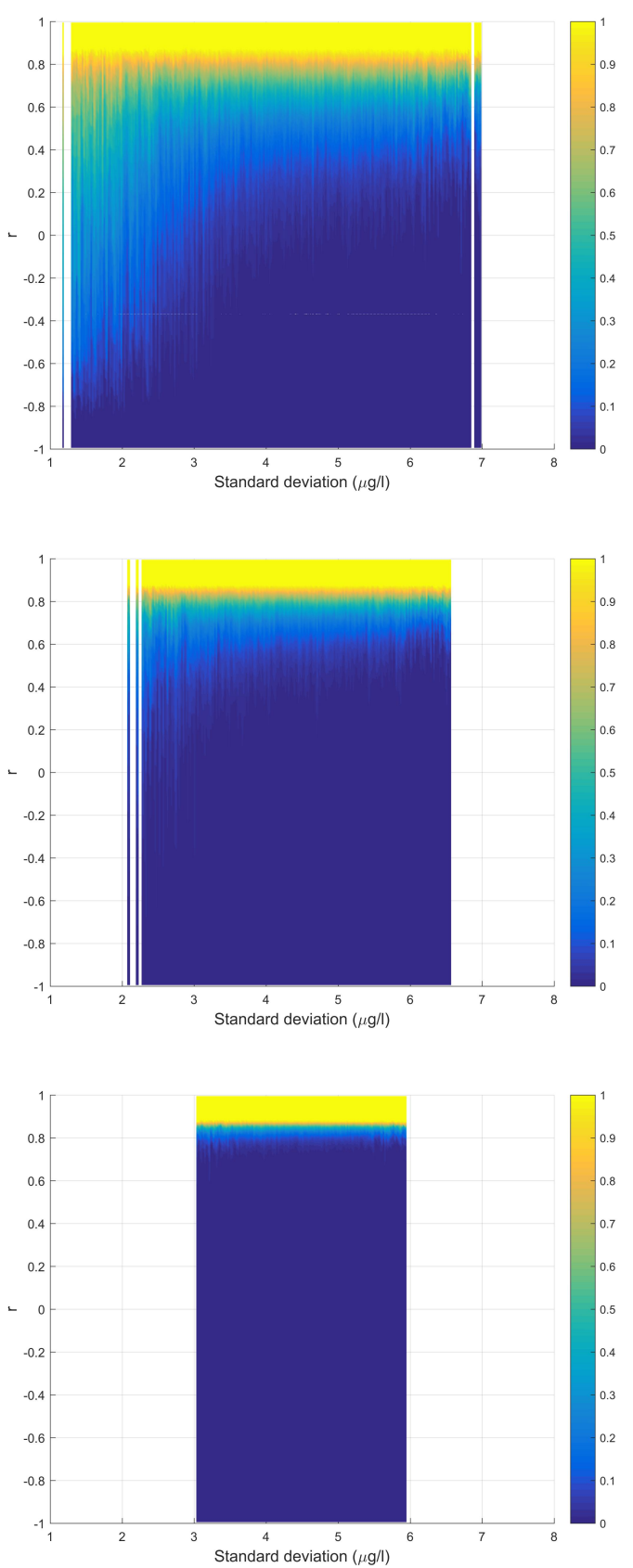

Figure 7. Cumulative probability histograms derived using 5 bands and 8 (top), 12 (middle) and 30 (bottom) randomly selected reference samples. The standard deviation bins for which the sum of counts is below 30 are rejected from probability histograms. 

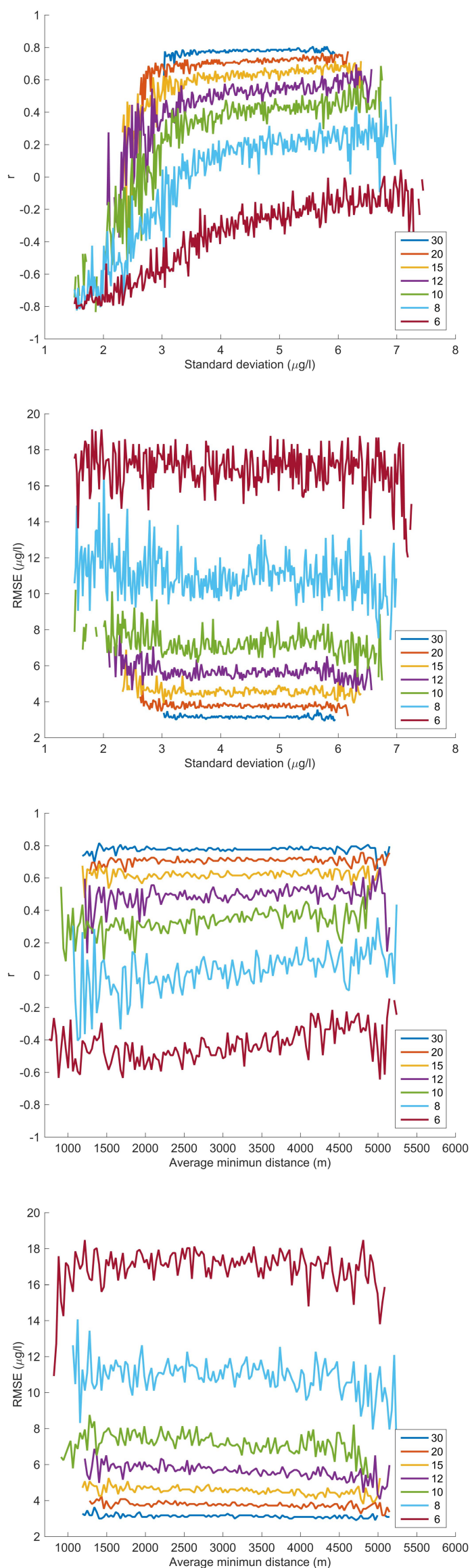

Figure 8. Correlation coefficient $r$ (top and second from bottom figures) and RMSE (second from top and bottom figures) for different number of randomly selected measurements. All figures: 50000 random selection, probability level 0.05 and 5 bands.
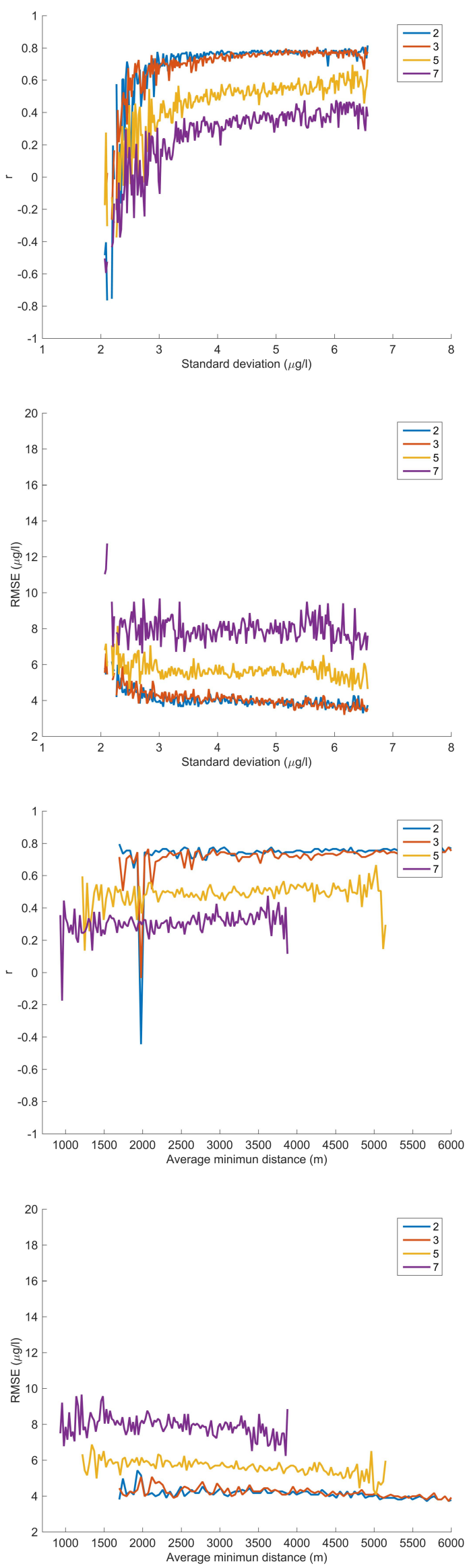

Figure 9. Correlation coefficient $r$ (top and second from bottom figures) and root mean square error (RMSE) (second from top and bottom figures) for different number of wavelength bands as variables. All figures: 12 randomly selected reference samples, 50000 random selection and probability level 0.05 . 

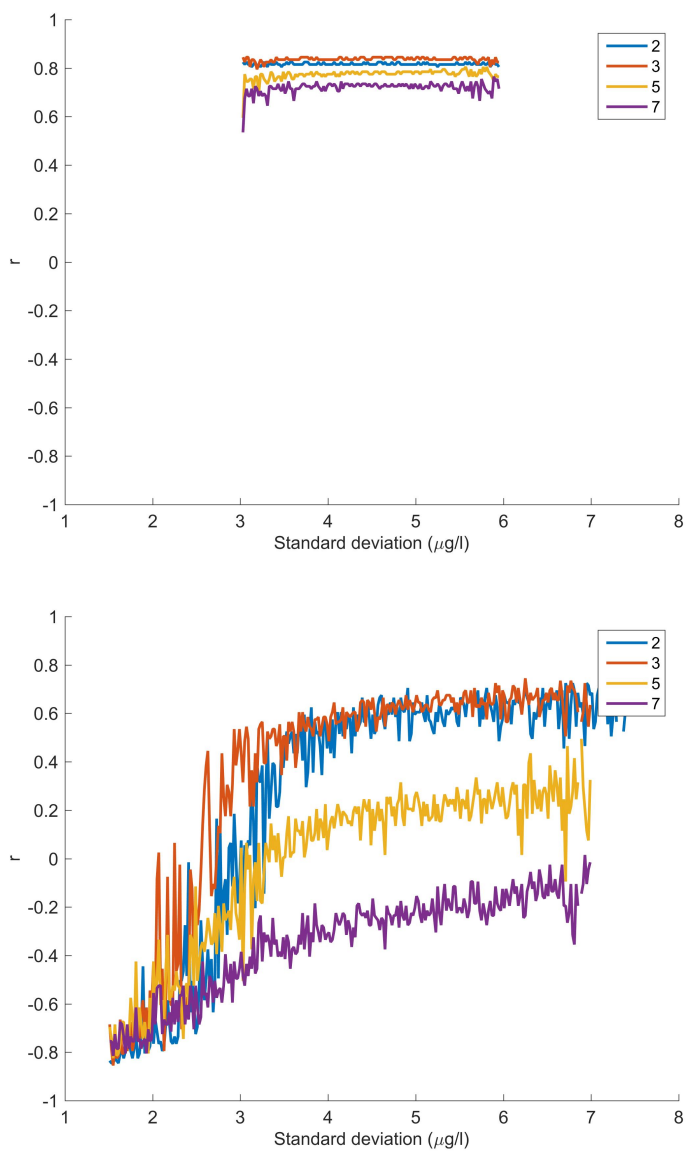

Figure 10. Correlation coefficient $\mathrm{r}$ for different number of wavelength bands as variables. 30 (top) and 8 (bottom) randomly selected reference samples. Both figures: 50000 random selection and probability level 0.05 .
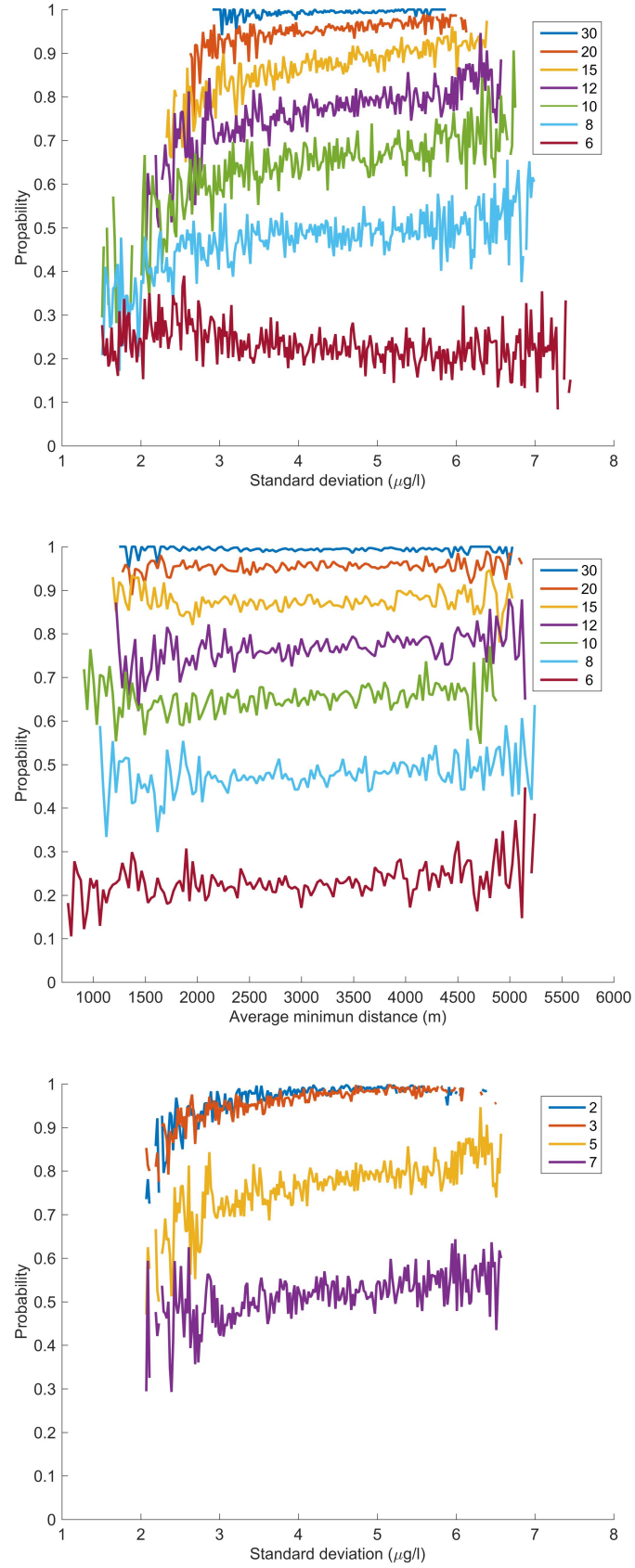

Figure 11. Probability that the correlation coefficient $r>0.7$ for different number of randomly selected samples (top and middle figures) and number of wavelength bands used as variables (bottom). 5 bands used for top and middle figures and 12 randomly selected reference measurements for bottom figure results. 
The International Archives of the Photogrammetry, Remote Sensing and Spatial Information Sciences, Volume XLII-3/W3, 2017

Frontiers in Spectral imaging and 3D Technologies for Geospatial Solutions, 25-27 October 2017, Jyväskylä, Finland

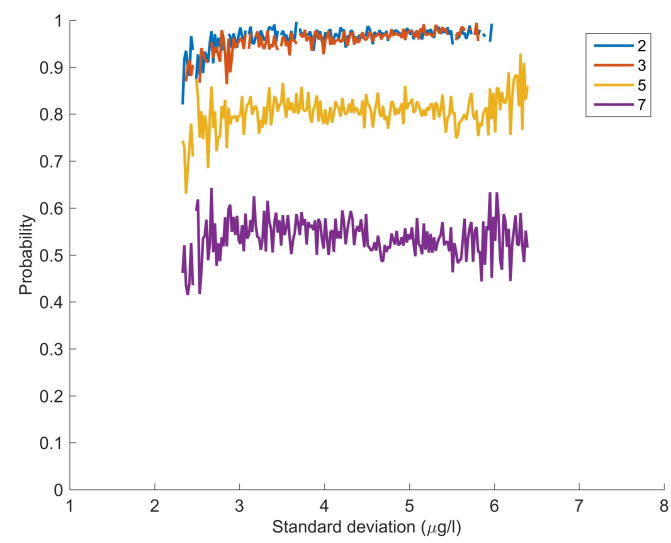

Figure 12. The probability that the RMSE $<4.0$ is presented for different number of wavelength bands used as variables. 12 randomly selected reference measurements. 